\title{
La seguridad, la higiene industrial y la protección del medio ambiente en la administración empresarial nicaragüense
}

Onassis Santiago Delgado Castro ${ }^{1}$

1 Maestría en Administración y Dirección de Empresas (MADE) Facultad de Ciencias Económicas y Empresariales de la UCA. Apdo. 69, Managua, Nicaragua.

Recibido: abril 2006 / Aceptado: mayo 2006

ESTE TRABAJO SE ENFOCA EN LA IMPORTANCIA DADA EN Nicaragua a la seguridad, la higiene laboral y la protección del medio ambiente. Se analiza el impacto de la industrialización, la globalización, las políticas de los organismos internacionales y su incidencia en la gestión empresarial; se presenta una visión general del marco legal vigente en Nicaragua respecto de las condiciones laborales y la protección del medio ambiente; se exponen los aspectos ético, cultural, funcional y organizacional de la empresa y la importancia de implantar un sistema de seguridad, higiene y protección ambiental para aplicar cualquiera de las estrategia genéricas descritas por Michael E. Porter, liderazgo en costos, diferenciación o enfoque. Se analizan los cinco factores de competitividad y el correspondiente análisis de fortalezas, oportunidades, debilidades y amenazas (FODA) que impactan sobre los sectores industriales de Nicaragua. Sobre esta base, se proponen lineamientos que las empresas nicaragüenses deberían adoptar.

Palabras clave: seguridad industrial-legislación-Nicaragua, salud ocupacionalNicaragua, protección del medio ambiente, competitividad

\section{Introducción}

Actualmente, para que una empresa sea competitiva nacional e internacionalmente, además de ser rentable, tiene que tener una ética empresarial expresada en valores y políticas de obligado cumplimiento y que cumpla con las normas de seguridad, higiene industrial y protección del medio ambiente. Este trabajo pretende a proponer lineamientos relacionados con este asunto que se puedan implantar, como parte de la gestión gerencial, en las empresas nicaragüenses, sirviendo de base para reformular la 
actual estrategia competitiva.

Muchas empresas han enfrentado demandas por violaciones a las normas de seguridad y daños ocasionados al ambiente, lo que ha deteriorado su imagen ante los consumidores. El objetivo de las empresas es incrementar su valor a largo plazo, maximizando sus utilidades. Pero este planteamiento está siendo cuestionado por un enfoque ético que tiene como precepto la seguridad, la higiene industrial y la protección del medio ambiente.

Se espera que este trabajo aporte al país la promoción del cambio hacia esa nueva cultura empresarial, motive a gerentes y empresarios a establecer estrategias competitivas que incluyan la observancia de la seguridad, higiene laboral y protección del medio ambiente porque los lineamientos presentados son pertinentes a las estrategias básicas y aplicables al mercado nacional, a las funciones de seguridad e higiene industrial en la estructura organizativa de las empresas, y a los requerimientos legales vigentes en Nicaragua.

\section{Impacto de la industrialización}

La industria es un conjunto de operaciones y procesos tecnológicos que posibilitan la transformación de los recursos naturales o de productos semielaborados, en otros que satisfagan las demandas de un grupo de personas, de otras empresas o de un mercado específico. Tras la revolución industrial se experimentaron entre otras cosas:

- Incremento de la producción.

- Mejoras organizativas, que parten de la división del trabajo, la especialización y 1 producción en cadena.

- Reducción de costos de producción, producción estandarizada y masiva y producción en serie.

- Incremento del consumo y de la demanda, reducción de precios de los productos e incremento de la competencia.

Pero también:

- Desempleo que abaratara la mano de obra.

- Aumento de la brecha económica entre países y a lo interno de cada país.

- Mayor exposición de los trabajadores a riesgos y peligros inherentes al nuevo ambiente de trabajo.

- Contaminación de la atmósfera, las aguas y los suelos.

Aunque de manera muy incipiente, la industrialización ha impactado a Nicaragua. Por lo tanto, es hora de incluir en la visión y misión de las empresas el respeto y protección del medio ambiente y la mejora de las condiciones laborales.

\section{Impacto de la globalización}

La globalización es la facilidad de movilización de capitales de una plaza a otra y el incremento del comercio internacional entre países. Actualmente, la globalización es una realidad con la que hay que vivir o a la que hay que sobrevivir. En el proceso de globalización, compiten empresas, organizaciones, y corporaciones mundiales para obtener los beneficios 
de la estrategia global para ser más competitivos que el oponente.

Hay una estrecha relación entre industrialización, globalización y riesgo. La influencia de la globalización en la seguridad, higiene industrial y protección del medio ambiente está intrínsecamente ligada con la globalización del riesgo, con dos vertientes:

- Efectos transnacionales, como los efectos de accidentes relativos a la explotación de fuentes nucleares (caso Chernóbil, Abril 1986); el "efecto invernadero" de alcance mundial; la destrucción de la capa de ozono, etc.

- La globalización económica y de intercambios comerciales implica un proceso de transferencia de fuentes de peligro: la carne de las "vacas locas"; propagación de enfermedades como el dengue o el SIDA, a regiones donde antes no existían; etc.

En estas condiciones: ¿vale la pena participar de la globalización? Definitivamente, sí.

- $\quad$ Porque significa vincularse con el potencial alentador que conlleva.

- Porque es un proceso indetenible.

\section{Organismos internacionales, seguridad laboral y protección del medio ambiente}

A medida que el mundo se globaliza, los organismos internacionales influyen más sobre los países miembros en su actividad económica, política y social, pero también sobre la seguridad, la higiene laboral y la protección del medio ambiente.

Estos organismos han incorporado requerimientos de protección ambiental para perseguir un desarrollo económico sostenible en los países beneficiados y asegurarse un mejor futuro común, de forma que el financiamiento de los proyectos de desarrollo incluyen estudios previos de impacto ambiental.

El Programa de las Naciones Unidas para el Desarrollo (PNUD), en su estudio del desarrollo humano en Nicaragua 2000, expresa que el desarrollo sustentable se basa en comprender mejor la diversidad de los ecosistemas, brindar soluciones localmente adaptadas a los problemas del medio ambiente, y vigilar mejor las consecuencias del desarrollo sobre el ambiente.

Igualmente, en el ámbito del tratado del Área de Libre Comercio de las Américas (ALCA), del cual Nicaragua es participante, los dos últimos objetivos de las negociaciones son:

A. Procurar quelas políticas ambientales y de liberalización comercial se apoyen mutuamente, tomando en cuenta los esfuerzos emprendidos por la OMC y otras organizaciones internacionales.

B. Asegurar, de conformidad con las respectivas leyes y reglamentos, la observancia y promoción de los derechos laborales, renovando los compromisos de respetar las normas fundamentales del trabajo internacionalmente reconocidas, y reconociendo que la Organización Internacional del Trabajo es la entidad competente para establecerlas. 


\section{La seguridad e higiene laboral}

El Consejo Nacional de Higiene y Seguridad se creó el 18 de agosto de 1993, por Decreto 190 del 25 de abril de 1993 y se declara el 18 de agosto Día Nacional de la Seguridad, Higiene y Salud de los Trabajadores. Además, en diversas instituciones se ofrecen diplomados en Higiene y Seguridad Industrial, que debieran mejorar los conocimientos de quienes manejen los programas de higiene y seguridad.

Pero según registros del Ministerio del Trabajo, en 1999 ocurrieron 46 accidentes laborales mortales; 776 graves y 15 muy graves. Estas cifras corresponden a los accidentes reportados. Los sectores más susceptibles de accidentes laborales son el eléctrico y el industrial.

Por otra parte, muchos empleadores violan las disposiciones legales del Código del Trabajo en materia de pago de prestaciones sociales, salarios y cumplimiento de los reglamentos de higiene y seguridad del trabajo. En consecuencia, los obreros son afectados por falta de equipos de protección personal y por desconocimiento.

Asimismo, muchos trabajadores padecen enfermedades ocupacionales. También se maltrata verbal, psicológica y hasta físicamente en algunas empresas. Según una encuesta del Movimiento de Mujeres Desempleadas "María Elena Cuadra", sobre una muestra de mil 500 trabajadores encuestados, el 74\% no ha recibido capacitación sobre los riesgos inherentes a su trabajo y el 61\% no tiene equipo de protección personal.

Sin embargo, la mejora en la seguridad reporta beneficios económicos, pues los accidentes tienen consecuencias económicas a las empresas e impactan en las relaciones laborales. Una dificultad surge porque los accidentes se conciben como causados por la fatalidad, pero se deben a la falta de análisis del riesgo en el trabajo. Además, se cree que basta con tener procedimientos específicos para evitar accidentes laborales; pero también es necesario aplicarlos.

En 2001, el INSS reportó 15,296 accidentes y enfermedades ocupacionales en Nicaragua, versus 12,830 reportados en el año 2000. Las actividades económicas que más riesgo reportan son los servicios sociales, fabricación de textiles, fabricación de prendas de vestir y de productos alimenticios; en cuanto al sector agroindustrial, se deben a la introducción de tecnología mecanizada e intoxicación por sustancias químicas.

Hay diferencias entre los datos del MITRAB y del INSS, lo que confirma que no todos los accidentes son reportados al MITRAB, porque quien reporta al MITRAB es el empleador; en cambio, quien reporta al INSS es el empleado, pues necesita recibir atención médica.

Según el Banco Central de Nicaragua (BCN), el PIB incrementó, en millones de córdobas de 1980 , en $4.6 \%$ en $1996 ; 5.1 \%$ en $1997 ; 7.4 \%$ en $1999 ; 5.5 \%$ en el $2000 ;$ y 3\% en el 2001. Simultáneamente, el número de trabajadores se incrementó de 1,537,000 en 1996 hasta 1,900,400 en el 2001. Esto representa un incremento total de empleados en ese período de 363,400 (72,680 empleados anualmente) y equivale a un incremento total del 23.6\% y a un incremento anual promedio del 4.7\%. De la combinación de estadísticas del BCN y el INSS se 
deduce que si en el año 2001 se emplearon 1,900,400 nicaragüenses y se reportaron 15,296 accidentes, ocho de cada 1,000 nicaragüenses se accidentan en sus labores en un período de un año (casi el 1\%).

\section{Protección del medio ambiente}

La Ley 217 del Medio Ambiente y los Recursos Naturales señala que el ambiente es patrimonio común de la nación y constituye la base para el desarrollo sostenible del país. Por lo tanto, es deber del estado y de todos los habitantes proteger los recursos naturales y el ambiente, restaurarlos y procurar eliminar los patrones de producción y consumo no sostenibles.

El uso actual de los recursos naturales determina el futuro de Nicaragua. El desarrollo económico es sostenible cuando la actividad productiva respeta al medio ambiente. Los recursos vivos de Nicaragua están conformados por:

- 9 regiones ecológicas

- 2.2 millones de hectáreas en áreas protegidas.

- 9,000 especies (46 endémicas) de plantas vasculares.

- Más de 400 especies de plantas útiles y cultivadas.

- 251 especies de mamíferos.

- 676 especies de aves.

- 172 especies de reptiles.

- 62 especies de anfibios.

- 45 especies de peces de agua dulce.

Los suelos planos arables cubren el 11\% del territorio. Entre 1960 y 1998, unos 6.3 millones de hectáreas, anteriormente cubiertas por vegetación natural, se incorporaron a la agricultura y la ganadería; y la cobertura boscosa bajó de ocho a cuatro millones de hectáreas en ese lapso de tiempo. De continuar con ese ritmo de destrucción de bosques, Nicaragua podría perder su potencial agropecuario. Para mantener y recuperar la capacidad productiva es necesario ampliar el marco de la legislación ambiental; promover un ordenamiento en la tenencia y uso de la tierra; aplicar políticas y mecanismos que promuevan la transformación tecnológica de las fincas; promover planes y programas de ordenamiento territorial que permitan explotar la tierra según su vocación.

En cuanto al manejo del agua, es necesario ordenar su uso mediante una planificación maestra que dicte las regulaciones de gestión para los diferentes usuarios, para asegurar la recarga de los acuíferos superficiales y subterráneos, detener y revertir la contaminación de las aguas, proteger la biodiversidad acuática y conservar el potencial energético, turístico y recreativo.

Respecto de los desechos sólidos, todo indica que en el futuro se generarán más por lo que es necesario: reducir la cantidad de materiales arrojados en vertederos; reciclar los desechos; lanzar campañas educativas sistemáticas que motiven a la población a disponer adecuadamente su basura y mantener limpia la localidad; adoptar tarifas con las cuales los sectores de mayores ingresos, las zonas comerciales y las industrias soporten una parte del costo de los servicios a los sectores pobres; implementar soluciones para eliminar los 
desechos peligrosos domésticos, agrícolas, industriales y hospitalarios.

\section{Marco jurídico de la seguridad e higiene laboral}

En 1996 se promulgó un nuevo Código del trabajo, Ley 185, pues el de 1945 había sido superado. En él se incluyen regulaciones claras sobre la higiene y seguridad ocupacional y los riesgos profesionales. Posteriormente, el Código se ha complementado con 19 normativas sobre Higiene y Seguridad Laboral.

Además, Nicaragua es miembro fundador de la Organización Internacional del Trabajo (OIT). De esta membresía nacen derechos y obligaciones entre la OIT y el estado de Nicaragua. En los estados miembro se instituye una relación entre los empleadores, las organizaciones de trabajadores y el gobierno, denominada "Tripartismo" ( Empleadores-TrabajadoresGobierno).

Como consecuencia de esta relación, Nicaragua está incluida en el Proyecto Regional de

Seguridad y Salud Ocupacional para Centroamérica, Belice, Panamá y República Dominicana, cuyo compromiso es trabajar con gobiernos, empresarios y trabajadores de Centroamérica para abordar las necesidades críticas de mejoramiento de la seguridad y salud laboral en la región. La meta de este proyecto de dos años es reducir la incidencia de accidentes, lesiones y enfermedades laborales en los países integrantes.

\section{Marco jurídico del Medio Ambiente}

El artículo 60 de la Constitución reconoce el derecho a vivir en un ambiente saludable y el deber de preservarlo, conservarlo y rescatar el medio ambiente y los recursos naturales. Otros artículos relacionados con el Medio Ambiente son el 89, 102, 128, 177 y 181. En la década de los 90, se firmaron y ratificaron los instrumentos y declaraciones de la Cumbre de Río y a nivel Centroamericano: el Convenio de Biodiversidad, de Cambios Climáticos, de Plantaciones Forestales, etc.

En 1996 se aprueba la Ley 217, del Medio Ambiente y los Recursos Naturales que establece las normas para la conservación, protección y restauración del Medio Ambiente y los Recursos Naturales que lo integran, asegurando su uso racional y sostenible, de acuerdo a lo señalado en la Constitución.

Esta ley tiene como objetivos: prevención; promoción de la función social-ambiental de la propiedad; explotación racional de los recursos naturales; utilización correcta del espacio físico (ordenamiento del territorio); creación del Sistema Nacional de Áreas Protegidas; garantizar el manejo adecuado de las cuencas; la educación ambiental y el derecho a la participación ciudadana.

Nicaragua es también signataria de diferentes Convenios Internacionales relacionados con el Medio Ambiente, entre ellos: Convención sobre la Diversidad Biológica; Convenio de Basilea sobre el Control de Movimientos Transfronterizos de los Desechos Peligrosos y su Eliminación; Convenio de Estocolmo sobre los Contaminantes Orgánicos Persistentes (COP); 
Convenio de Viena y Protocolo de Montreal para la Protección de la Capa de Ozono.

\section{Estrategias competitivas}

Las empresas deben monitorear cuatro entornos íntimamente relacionados: el macroentorno (aspectos demográficos, macroeconómicos, regulaciones gubernamentales, etc.); el entorno industrial (estructura de la industria, forma en que se financia, productos típicos, etc.); el entorno competitivo (perfiles del competidor, modelos de segmentación, investigación y desarrollo, etc.); y el entorno interno de la organización (estructura de la compañía, su cultura, sus fortalezas y debilidades).

La intensidad de la competencia de una industria depende de cinco fuerzas que combinadas, determinan el potencial de un sector industrial:

- Riesgos de nuevas empresas o entrada de nuevos participantes.

- Intensidad de la rivalidad de los competidores actuales.

- Presión de productos sustitutos.

- Poder de negociación de los compradores.

- Poder de negociación de los proveedores.

También se agrega la fuerza del gobierno. Cada empresa merece un análisis de las cinco fuerzas que determinan la intensidad de la competencia; sólo después se pueden determinar las fortalezas y debilidades de la empresa en relación con la industria, y las oportunidades y amenazas que se le manifiestan en el entorno. Una buena estrategia emprende acciones ofensivas y defensivas para lograr una posición defendible contra las cinco fuerzas.

\section{Liderazgo en costos}

Esta estrategia consiste en alcanzar el liderazgo en costos globales mediante un conjunto de políticas funcionales encaminadas a éste objetivo y exige:

- Construcción agresiva de instalaciones de escala eficiente.

- Búsqueda vigorosa de reducción de costos a partir de la experiencia.

- Control riguroso de gastos variables y fijos.

- Evitar las cuentas de clientes menores y minimizar los costos en áreas de investigación y desarrollo.

- Fuerzas de ventas, publicidad y otras.

En toda la estrategia, los costos, que están íntimamente ligados con la calidad, el servicio y otras claves del éxito, son el tema central. Un principio básico en la operatividad de una organización es que la falta de planificación y organización salen muy caros. Además, la inseguridad y la falta de condiciones laborales hacen incurrir en pérdidas materiales, de tiempo y recursos humanos, que redundan en pérdidas financieras e incremento de los costos.

\section{Diferenciación}

Esta estrategia genérica persigue diferenciar el producto creando algo que se perciba 
como único. Actualmente, los consumidores buscan productos que no afecten a la salud, no contaminen, no destruyan la capa de ozono, no incrementen los gases con efecto invernadero, que se elaboren mediante procesos amables con el ambiente, que no produzcan residuos peligrosos, que los empaques puedan ser reciclados, que no destruyan la flora y la fauna.

Los negocios giran en torno a los deseos de los consumidores, quienes marcan las pautas para la producción de bienes y servicios. A medida que las personas se hacen más conscientes de que se deben cuidar de los productos que contienen sustancias peligrosas, o que deben de proteger el medio ambiente, surge la oportunidad de desarrollar productos con nuevos valores.

La gama de estos valores cada día se amplía más y toca aspectos tales como: trabajo infantil, condiciones laborales, discriminación racial, discriminación de género, sistemas políticos totalitarios, bajos salarios, impacto sobre la salud de los trabajadores, accidentes laborales, contaminación, destrucción de fauna y flora, etc.

Un ejemplo: en los 80, la marca "Niké" fue acusada de obtener ganancias onerosas pagando salarios bajísimos a trabajadores asiáticos, en condiciones de trabajo deplorables. Incluso algunas revistas, en los Estados Unidos de América, instaron a rechazar sus zapatos. Esto afectó la imagen de esta empresa y disminuyó sus ventas. ¿Qué le ocurriría a una pequeña empresa nicaragüense que intente ingresar a un mercado exigente, si le señalan como responsable de contaminación?

La estrategia de diferenciación de un producto puede capitalizar el sistema de seguridad, higiene y protección ambiental:

- Se produce a través de procesos "limpios".

- Es natural y no contamina el ambiente.

- El empaque es reciclable o biodegradable.

- Imágenes de publicidad que evoquen el respeto al medio ambiente.

- Uso de la frase "hecho bajo las más estrictas normas de higiene y calidad."

- Producto de uso seguro.

\section{La ética y los valores en la empresa}

La ciencia y tecnología son manifestaciones humanas extraordinarias. Pero el desarrollo tecnológico encierra graves peligros si no le acompaña una ética de los valores que dé sentido humano a los avances. La ética es el único medio para contemplar el actual desarrollo tecnológico sin degradarlo porque, aunque la ciencia hace progresar al hombre, no lo vuelve más humano. Por el contrario, puede deshumanizarlo si una ética no encauza los avances.

La Seguridad, la Higiene Industrial y la Protección del Medio Ambiente constituyen un problema ético, considerando la ética como un conjunto de reglas de comportamiento y formas de vida, a través de los cuales tiende el hombre a realizar el valor de lo bueno. Y se consideran valores las cualidades que poseen las personas o grupos. Cada valor tiene siempre un antivalor; además, los valores son mudables dependiendo del tiempo y el lugar. 


\section{Ética empresarial}

Se define la ética empresarial como el código interno de cada compañía, por el que se establece un marco jurídico propio, escrito o consuetudinario, por el que se autorizan o sancionan determinadas conductas. Ese código interno se expresa en políticas que se transforman luego en normas y reglamentos.

La ética empresarial concuerda con la ética de su entorno, pues las empresas no son islas e interactuan con otras empresas, la comunidad y el gobierno. De esta manera, si uno de los valores de la empresa es la justicia, la ética empresarial, que es el conjunto de normas a través de las cuales realiza sus negocios, debe estar en armonía con el cumplimiento de las leyes del país.

La implantación, aunque sea paulatina, de códigos de ética empresarial en las empresas de Nicaragua sería un poderoso factor de cambio en la economía nicaragüense. Por mencionar algunos problemas de ética empresarial que tendrían que ser evitados: el fraude y la corrupción; evasión fiscal; publicidad engañosa; venta de productos vencidos; omisión de información de efectos perjudiciales del producto; contaminación del ambiente; condiciones inseguras de trabajo; pago de salarios inferiores al salario mínimo, ya no se diga los que no cubren ni las necesidades básicas; no pagar deudas o erogaciones; asumir compromisos financieros imposibles de realizar; declaraciones falsas de propiedad; estelionato y hurto; modificación de los registros financieros con fines ilegales; etc. Cada una de estas acciones trae consigo sus consecuencias y penalidades legales.

\section{Cultura y valores organizacionales}

La cultura es un conjunto de creencias y expectativas que mantienen en común, y profundamente, los miembros de una comunidad. Los valores empresariales originan normas situacionales, "la forma como hacemos las cosas aquí", que se evidencian en el comportamiento observable. Este comportamiento normativo se convierte en la base para la validación de las creencias y los valores originan las normas. El circuito cerrado creenciasvalores-normas-creencias constituye el proceso de desarrollo cultural al que se atribuye la tenacidad de las culturas.

En consecuencia, para establecer una nueva cultura de seguridad, higiene y protección ambiental dentro de la organización, como parte de un plan estratégico para asegurar competitividad, se debe trabajar en las creencias, en los valores y en las normas. Un individuo que cree que no es necesario usar el equipo de seguridad, o que no es importante cumplir con los procedimientos y medidas de seguridad, manifiesta falta de cultura organizacional de seguridad.

Una organización es un sistema social con límites relativamente establecidos, creado deliberadamente con carácter permanente para el logro de una finalidad, que combina recursos humanos y materiales, cuya esencia es la división del trabajo y la coordinación, y que implica unos procesos intraorganizativos e interorganizativos y unos valores. 
Las organizaciones están compuestas por personas que, en el ejercicio de sus funciones, aportan sus propios valores. Por lo tanto, los valores empresariales y organizacionales son el resultado de la interacción o sinergia de valores y podría darse el caso de que en una empresa existan políticas y normas ajustadas a ciertos valores y también es posible que el comportamiento de sus miembros difiera, porque no las comparten.

Los valores organizacionales son características terciarias; no son visibles ni tangibles, y sólo son observables a través del comportamiento de sus miembros. Por ello, implantar un cambio de cultura organizacional, es una tarea compleja, que enfrenta la resistencia al cambio que presentan los miembros de una organización.

\section{Ética y rentabilidad}

La contribución primordial de las empresas a la sociedad es su eficacia para transformar recursos y distribuirlos como productos. En este proceso se toman decisiones de negocios de acuerdo a un código de ética empresarial que se basa en valores; por lo tanto, todas las decisiones organizacionales tienen como fundamento los valores.

Por otra parte, el objetivo de toda organización empresarial es generar utilidades de forma rentable, esto es incrementar su valor de manera sostenible a lo largo del tiempo. Entendiendo como "valor", el valor presente de los flujos de efectivo de la empresa esperados en el futuro. Dicho de otra forma, el objetivo es maximizar los beneficios.

Hace más de 200 años el economista inglés, Adam Smith, escribió en su libro, "La riqueza de las naciones (1776)": No es la benevolencia del carnicero, del cervecero o del panadero la que nos procura el alimento, sino la consideración de sus propios intereses.

Es decir: las empresas existen sobre todo porque han demostrado ser mecanismos útiles para la producción y distribución eficaz de bienes y servicios; además, las empresas miran en las necesidades insatisfechas de un mercado una oportunidad de negocio, considerando sus propios intereses.

De hecho el énfasis estratégico de las empresas ha evolucionado. Al principio, se ponía el énfasis en el producto; luego, en los procesos tecnológicos; después, en la calidad total; y por último, en la satisfacción del cliente. Posiblemente se seguirá evolucionando, pero el objetivo es el mismo: maximizar los beneficios.

Surge entonces la pregunta: la ética empresarial, ¿es una limitante de la rentabilidad o de la oportunidad de negocios?

En realidad la rentabilidad y la oportunidad de negocios tienen límites: Llega un punto en que es muy difícil reducir el costo de producción sin detrimento de la calidad; diferenciar un producto por precio tiene un límite, que es la elasticidad de precio, el empuje de los competidores y las leyes de oferta y demanda del mercado. En el caso de la oportunidad de negocios hay límites como las leyes civiles y mercantiles; la propia salud y resistencia física y psíquica; hasta los horarios de trabajo y las relaciones familiares que, en determinado 
momento, pudieran imponer un costo de oportunidad emocionalmente superior.

Desde ese punto de vista podría decirse que la ética es una limitante, ya que define normas y reglas de comportamiento, limitando el radio de acción para los negocios. Por ejemplo, no se permite el soborno. Pero lo característico de los principios éticos es evitar consecuencias indeseables, tales como la pérdida de imagen de la empresa, multas, pagos de indemnizaciones e incluso cierres, y está dirigida u orientada a conseguir las mejores actuaciones posibles. Entonces la ética persigue la laboriosidad, el orden, la confianza, la disciplina, la sintonía para trabajar en equipo, cumplimiento de las leyes; valores que, en el fondo, no representan limitantes sino factores útiles para lograr ventajas competitivas.

Puesto que el código de ética empresarial en el mundo actual y por ende en Nicaragua exige la seguridad, higiene industrial y la protección del medio ambiente, cada empresa debe expresar sus principios y valores éticos relativos a este tema a través de políticas, que deben ser comunicadas a todos los empleados para su observancia. A la luz de estas políticas deben girar las actividades y negocios que se realizan, y los procedimientos deben ser elaborados en observándolos estrictamente.

\section{Cultura organizacional}

Los recursos humanos son los más importantes de una empresa. Por lo tanto, es necesario cuidarlos, darles mantenimiento y desarrollarlos. Esa responsabilidad corresponde al sistema de higiene, seguridad y protección personal, que debe ser adoptado como una necesidad básica. Por lo tanto, es necesario un cambio de mentalidad que genere una nueva forma de hacer y gerenciar negocios, mediante:

- Reconocer que el capital humano empresarial es un elemento indispensable.

- Reconocer que la seguridad laboral es una necesidad básica de los individuos.

- Reconocer la importancia de estas funciones en la organización.

\section{Importancia del capital humano}

Actualmente, el capital humano se conceptúa como inherente a la empresa. Sin embargo, el empresariado nicaragüense dedica más esfuerzo al mantenimiento de equipos yherramientas de trabajo, que al trabajador, de cuyo desempeño depende el uso de los recursos materiales, la eficiencia y la eficacia de las operaciones. De esta forma, es común asignar recursos al mantenimiento de equipos y no a la capacitación, desarrollo del trabajo en equipo, mejora de las condiciones laborales, diseño de instalaciones más seguras, etc.

En Nicaragua abunda la mano de obra no calificada, lo que constituye un reto a las empresas para que sus negocios sean competitivos. Los bajos salarios, la carencia de una cultura de trabajo con aceptables estándares de seguridad, y las malas condiciones laborales son una barrera a superar en el medio empresarial. En consecuencia, los empresarios nicaragüenses y el gobierno tienen que reconocer la importancia de los recursos humanos en las empresas y propugnar por una mayor inversión para su desarrollo y protección, igual que los demás activos. 


\section{La pirámide de Maslow}

La motivación impulsa al ser humano a actuar de determinada manera o, por lo menos, origina una propensión hacia un comportamiento específico. Maslow considera que las necesidades del hombre son crecientes a lo largo de su vida y a medida que el hombre satisface sus necesidades básicas, otras más elevadas ocupan el predominio de su comportamiento. Maslow presenta la siguiente jerarquía de necesidades, de configuración piramidal:

Necesidades fisiológicas: alimento, agua, aire y descanso.

Necesidades de seguridad: protección contra el peligro o las privaciones. Incluye la necesidad de seguridad en los sentidos físico y psicológico. Necesidades sociales: amistad, pertenencia a grupos, etc. Supone la necesidad de atención y aceptación social.

Necesidades de autorrealización: realización potencial, utilización plena de los talentos individuales. Es la forma suprema de motivación, de ser mejor en todo sentido.

Una necesidad satisfecha no origina ningún comportamiento; sólo las necesidades no satisfechas influyen en el comportamiento y lo encaminan hacia el logro de objetivos. En la medida que el individuo controla sus necesidades fisiológicas y de seguridad, aparecen lenta y gradualmente necesidades más elevadas; sociales, de estima y autorrealización. Cuando el individuo satisface sus necesidades sociales, surgen las necesidades de autorrealización; esto significa que las necesidades de estima son complementarias de las necesidades sociales, en tanto que las de autorrealización lo son de las de estima. Los niveles más elevados de necesidades, de acuerdo a Maslow, sólo surgen cuando los niveles más bajos han sido alcanzados por el individuo.

Una forma efectiva de promover la seguridad en una empresa es usando la motivación que impulsa al personal a alcanzar objetivos y adoptar actitudes positivas ante la seguridad, porque la considera beneficiosa.

Para motivar al personal es necesario hacer un diagnóstico de sus necesidades, especialmente de quienes por su trabajo corren mayores riesgos.

\section{Funciones de la Seguridad, Higiene Laboral y Protección Ambiental}

La higiene laboral es de carácter preventivo y evita que el trabajador se enferme o se ausente de forma provisional o definitiva del trabajo eliminando las causas de enfermedad profesional; reduciendo los efectos perjudiciales provocados por el ambiente de trabajo en personas con capacidades diferentes; manteniendo la salud de los trabajadores; y aumentando la productividad por medio del estudio y control del ambiente de trabajo.

La seguridad laboral es el conjunto de medidas técnicas, educacionales, médicas y psicológicas empleadas para prevenir accidentes, tendientes a eliminar las condiciones inseguras del ambiente, y a instruir o convencer a las personas acerca de la necesidad de implantación de prácticas preventivas. Contempla tres áreas: prevención de incidentes; prevención de robos; y prevención de incendios. 


\section{Comité de seguridad}

Adicional al departamento o sección de seguridad, higiene y protección personal, la gerencia debe nominar un comité de seguridad, que debe incluir a los gerentes de departamento y al director o gerente de la sección de seguridad. Este comité apoyará de forma directa e institucional, y en cada uno de los departamentos bajo su mando, los lineamientos, programas, normas y procedimientos de seguridad que con apoyo de la gerencia general impulsa el departamento de seguridad.

Adicionalmente, servirá para tratar asuntos claves de este tema en la empresa, y que por su naturaleza podrían requerir una toma de decisión importante, estudiar propuestas de modificación al presupuesto, la aprobación de proyectos relacionados con la materia y analizar propuestas de nuevos proyectos que puedan afectar la seguridad de la empresa 0 de sus procesos.

Todo recurso asignado a la higiene, seguridad y protección del medio ambiente, correctamente administrado, constituye una inversión y no un gasto, pues los beneficios superan los recursos invertidos.

\section{Compromiso y liderazgo gerencial}

El cumplimiento de las normas de seguridad en una empresa es inherente a los valores y normas éticas de la empresa; por lo tanto, el problema de la seguridad y la higiene es un problema de ética y valores. Es allí donde entra la responsabilidad de los directivos de la empresa en promover los valores y en conducir a la empresa dentro de sus políticas o normas de ética como una actividad intrínseca en el cumplimiento de la misión.

La organización, la administración y la dirección son los tres pasos que comúnmente se siguen para distribuir asignaciones entre el personal de la empresa, poner el trabajo en movimiento y definir responsabilidades y obligaciones a fin de generar orden y control. Cuanto mayor sea el número de personas que trabajan en una organización, tanto más importante es emplear un enfoque sistemático, pues un programa no es suficiente.

La seguridad se ha definido como una responsabilidad de cada uno de los miembros de la organización. Pero, dentro de la organización, esta definición podría ser engañosa pues la mayoría de las funciones de la sociedad moderna se cumplen a través de una jerarquía. Por lo tanto, la responsabilidad por la seguridad de otros aumenta a medida que ascienden los niveles jerárquicos.

Corresponde a los jefes de toda organización asegurar el mantenimiento de las instalaciones y proporcionar el ambiente idóneo para que el capital humano de la empresa ejecute su trabajo satisfactoriamente. Los dirigentes de una empresa deben estar conscientes de que las lesiones son costosas. No solamente se incurre en costos médicos e indemnizaciones, sino también de tiempo productivo y valioso y pueden producirse daños a la propiedad, por los que un administrador tiene que responder ante accionistas o dueños. Además, hay costos emocionales de gran alcance en la actitud de los trabajadores ante la empresa y el trabajo. 
Se espera entonces, que los dirigentes de las empresas nicaragüenses den los pasos necesarios, -por medio de la educación y capacitación de los trabajadores, estableciendo políticas y normas de seguridad, brindando las condiciones necesarias para una ejecución de trabajos segura-, para prevenir accidentes lamentables.

\section{La resistencia al cambio}

Las empresas, como todas las organizaciones sociales, son relativamente conservadoras y generalmente no están preparadas para enfrentar el cambio. Muchas circunstancias actuales están obligando a un cambio de cultura organizacional en relación con las condiciones laborales: el avance de la globalización, el empuje externo para establecer leyes que protejan los derechos de los trabajadores, las demandas de un mercado cada vez más consciente de los peligros de la contaminación y las condiciones laborales, los requerimientos de las organizaciones para brindar financiamiento, etc.

El proceso de cambio implica necesariamente la reformulación de conceptos, paradigmas y valores muy incorporados a la cultura de la organización y de sus dirigentes. Pero écómo lograr el cambio en el ámbito de la seguridad e higiene laboral?

La respuesta en manos de la dirigencia y se resuelve mediante acciones: mostrando compromiso con la seguridad e higiene industrial por parte de la gerencia; facilitando recursos humanos, materiales y financieros; creando un ambiente de trabajo seguro con diseños adecuados; seleccionando al personal adecuado; y estableciendo un programa de capacitación, que oriente hacia el cambio de cultura e indique sus beneficios.

\section{Áreas a ser desarrolladas en las empresas nicaragüenses}

- Visión y misión. Incluir la realización de negocios en un lugar seguro donde "nadie salga lastimado".

- Valores y cultura. Adoptar estos valores y crear una cultura organizacional en concordancia a sus principios.

- Gerencia. Mayor liderazgo y compromiso en esta materia.

- Condiciones laborales. Los empresarios y gerentes deben priorizar dar a los trabajadores un ambiente de trabajo sano y seguro.

- Sistema de Seguridad. Destinar recursos a la creación de un sistema de higiene y seguridad que sea parte de la estructura organizacional.

- Análisis de riesgo. Los encargados de seguridad e higiene de la empresa deben coordinar análisis de riesgos para mitigarlos.

- Personal y capacitación. Capacitar adecuadamente al personal para operar los equipos necesarios.

- Equipo de protección personal. Facilitar al personal los equipos de protección requeridos para trabajar con seguridad.

- Procedimientos y normas. Elaborar un manual de procedimientos y asegurar su conocimiento y uso.

- Investigación de accidentes. Investigar cada accidente laboral hasta encontrar sus causas y modificarlas. 
- Grupos de seguridad. Gestionarán la seguridad en sus áreas de trabajo identificando peligros y proponiendo soluciones.

- Motivación. Mejorar el "mercadeo" de la seguridad para mostrar los beneficios del cumplimiento de las normas.

\section{La Higiene Laboral}

La higiene ocupacional se define como la ciencia que investiga la prevención, reconocimiento, evaluación y control de los factores ambientales en el lugar de trabajo y que pueden causar enfermedades, deterioro de la salud e incapacidad entre los trabajadores y miembros de la comunidad. La higiene ocupacional determina los siguientes causales de enfermedades ocupacionales: tipo de agente; vía de entrada; tiempo de exposición; e intensidad de la exposición.

Los agentes que más afectan a los trabajadores son: ruidos; falta de ventilación; vibraciones; radiaciones, que pueden ser ionizantes (nuclear y rayos X) y no ionizantes (electromagnética, ultravioleta, infrarroja, las microondas y las ondas de radio, la radiación del radar, y el rayo láser); iluminación; temperatura; presión; agentes químicos; y ergonomía.

\section{Análisis de riesgos en las empresas}

Para analizar los riesgos, es necesario primero identificarlos, empezando por identificar los peligros y sus consecuencias. Peligro es un concepto diferente de riesgo. Peligro es cualquier condición existente que puede generar daños. Por ejemplo, un horno con carcasa metálica sin aislamiento es un peligro, pues puede provocar quemaduras que pueden ocasionar inhabilitación y muerte.

El riesgo, por su parte, tiene dos componentes: probabilidad y consecuencia. La probabilidad es un cálculo matemático, que puede estar basado en datos estadísticos, propios de la empresa, sectoriales o nacionales. Por ejemplo, la probabilidad puede calcularse a partir del número de accidentes ocurridos durante un lapso de tiempo en el país o en el sector.

La consecuencia tiene que ver con la gravedad del accidente y se mide por su impacto. Por ejemplo: ¿qué consecuencia tendría si un trabajador cae desde un lugar alto? ¿Qué impacto tendría sobre las operaciones de la empresa? El impacto puede ser financiero, a la imagen de la empresa, en la producción, sobre los activos o la propiedad y, por supuesto, impacto humano.

El análisis de riesgo se hace cuando se han identificado peligros que pueden acarrear consecuencias. Es parte de un estudio de identificación de procesos riesgosos en la planta. Entre ellos:

- Actividades donde hay conocimiento de frecuentes accidentes graves.

- Operaciones de grúas y montacargas.

- Levantamiento de objetos pesados con tecles.

- Trabajos en equipos o instalaciones de alto voltaje.

- Transporte de líquidos inflamables. 
- Manejo de materiales tóxicos o peligrosos.

- Trabajos sobre andamios, o en lugares elevados o altos.

- Ingreso a recipientes que contuvieron productos peligrosos.

- Soldadura en lugares cerrados.

- Lugares donde hay fuentes de alta temperatura.

El análisis de riesgos es un método de prevención de accidentes usado en la industria moderna y consta de los siguientes pasos:

1. Establecer el escenario.

2. Categorizar la probabilidad y la consecuencia.

3. Analizar el escenario y contestar a preguntas como: ¿Qué medidas pueden tomarse para evitar ocurra un accidente? Analizan las condiciones inseguras, reconocer y estudiar los elementos que entran en acción durante la ejecución de ese trabajo.

4. Hacer las recomendaciones correspondientes.

5. Definir las medidas mitigantes.

6. Comunicar el análisis de riesgo al personal pertinente.

\section{Investigación de accidentes}

El propósito de la investigación de accidentes es dejar en claro las causas que lo originaron para prevenirlos en el futuro. Se deben investigar los accidentes que involucran daños a la propiedad o instalaciones, los que implican primeros auxilios al personal, lesiones o fatalidades, contaminación, violación a las leyes del país, accidentes vehiculares, etc. Asimismo, las situaciones en que "casi” ocurrió un accidente.

Estadísticamente, en las industrias, por cada accidente grave, se dan 600 casos de "casi" accidentes, que constituyen una gran oportunidad aprender, especialmente porque no ocurrieron. La metodología de investigación de accidentes sigue los siguientes pasos:

1. Recolección de hechos.

2. Determinación de la causa del accidente.

3. Formulación de recomendaciones y propuesta de plan de acción.

4. Una vez aceptadas las causas, las recomendaciones y el plan de acción deben comunicarse al personal. El objetivo es que el accidente no vuelva a ocurrir.

\section{Capacitación del personal}

La experiencia en la industria demuestra que un gran número de accidentes se deben a la falta de capacitación de las personas directamente involucradas en determinada actividad:

- Desconocen de los peligros de la actividad.

- Desconocen de las medidas de seguridad.

- No saben identificar los peligros y riesgos en el área de trabajo.

- No han recibido entrenamiento suficiente para desempeñar el trabajo.

- Acaban de ingresar a la empresa y su experiencia es pobre.

- Fueron transferidos de puesto de trabajo dentro de la misma empresa.

Por ello, en la empresa debe existir un programa de capacitación para los empleados, 
que esté acorde con la complejidad técnica, conocimientos requeridos y niveles de riesgo del trabajo que realiza el empleado, para asegurar un buen desempeño. Los aspectos de seguridad deben ser parte de este plan o programa.

\section{La protección del medio ambiente}

El rol de la administración o gerencia en torno a este tema debe incluir:

- Estudios de impacto ambiental.

- Análisis de riesgos medioambientales.

- Auditorias internas relacionadas con la protección del medio ambiente realizadas periódicamente.

Esta gestión empresarial de protección del medio ambiente se puede combinar con aspectos estratégicos, que aseguran la competitividad, como:

- Mantener el primer puesto en el mercado.

- Producir lo mejor al menor coste.

- Sobrepasar las necesidades y expectativas de los clientes.

- Proteger y destacar la marca registrada y el valor de las acciones.

- Diferenciar sus productos y servicios.

- Reforzar los valores y la cultura de la organización.

\section{Estudio de Impacto Ambiental}

El estudio de impacto ambiental es parte del análisis de riesgo que las empresas deben realizar de acuerdo a su responsabilidad social y es uno de los más importantes en la gestión de protección del medio ambiente. La identificación, evaluación y jerarquización de los objetos de riesgo harán más visibles los peligros y más efectiva la protección a las personas, las propiedades y al medio ambiente.

Las personas responsables de tomar decisiones en las industrias deben reconocer la necesidad del manejo seguro de éstos riesgos atendiendo a las siguientes razones:

- La salud y seguridad de los trabajadores y vecinos de la empresa.

- Evitar daños a la propiedad, al medio ambiente y a los medios de producción de la propia empresa.

- La necesidad de mantener buenas relaciones con las autoridades y el público para su desarrollo positivo.

- La necesidad de una producción continua, mantener una entrega confiable y las buenas relaciones con los clientes y proveedores.

Los estudios de impacto ambiental están dirigidos tanto a los procesos presentes en la empresa y a los proyectos de modernización o de ampliación propuestos, y deben ser condición sine qua non para seguir operando en ese giro de negocio o de aprobar presupuesto de capital para proyectos, con la misma prioridad que la rentabilidad.

El gobierno de la República estimula las acciones a favor de la conservación del patrimonio natural y cultural de Nicaragua por parte de la ciudadanía. 


\section{Proyectos relacionados con la protección del medio ambiente}

En el caso del medio ambiente, el marco legal de Nicaragua introduce como variables importantes en el ámbito de negocio de las empresas, estándares y límites máximos permisibles de diversos parámetros.

En consecuencia, la instalación de una planta, su ampliación o la introducción de un nuevo proceso tecnológico, necesita ser estudiada a la luz de estas condiciones para asegurar su cumplimiento y evitar violaciones sanitarias, civiles, penales u otras.

Igualmente, las empresas actuales, si aparecen nuevas leyes y regulaciones que dejan obsoletos sus parámetros operativos (manejo de residuos líquidos, emisiones a la atmósfera, tratamiento de desechos, contaminación de las aguas, destrucción de la flora o fauna, probabilidad de daños a la comunidad, riesgo de la salud de sus empleados, etc.) deben alcanzar los estándares requeridos por el gobierno y entidades correspondientes o enfrentar las consecuencias, que podrían implicar multas, acciones para remediar daños, indemnizaciones, disminución de ventas e, incluso, cierre.

Para evaluar propuestas ambientalistas, que no implican incremento en la producción o flujos de efectivos positivos, sino que constituyen costos desde el punto de vista contable, no es adecuado aplicar el método de la TIR o el VAN.

De acuerdo a Gabriel Baca Urbina, en su libro "Evaluación de Proyectos", existen entre otros, dos métodos para evaluar estos proyectos:

1. El método de la razón Beneficio/Costo (B/C) que se utiliza para evaluar inversiones gubernamentales o de interés social. De este modo, no se cuantifican los beneficios y los costos, sino los criterios sociales. En este caso, de protección del medio ambiente.

2. El método del flujo anual (FA), que tiene los mismos principios que el VAN y la TIR. El Flujo anual (FA) se obtiene descontando todos los flujos de efectivos al presente y analizándolos a lo largo de todo el horizonte de planeación, es decir distribuyéndolos en cantidades iguales y equivalentes en todos los años de estudio.

Un ejemplo: hay que reducir las emisiones contaminantes de un equipo durante el proceso de producción, y hay varias alternativas para lograrlo. Dada su naturaleza, la realización de cualquier alternativa no incrementará la producción, pero traerá beneficios como no ser multados, no incurrir en gastos legales, en indemnizaciones o no sufrir cierres.

Entonces, para el análisis económico y selección, se usa el método FA, que hace una comparación de costos individuales entre las varias alternativas. Es decir, éste método permite analizar los costos en que se incurriría en cada alternativa, seleccionándose la que tenga menores costos.

Los proyectos relacionados con la seguridad, higiene laboral y protección del medio ambiente tienen un valor superior al financiero, que es el valor humanitario y ético. El compromiso gerencial con sus valores y la ética debe tener cabida en todos los ámbitos del negocio, incluidos los proyectos de corto, mediano y largo alcance. Una empresa que contamina el 
ambiente y no brinda las condiciones higiénicas y de seguridad laboral a sus empleados, no tiene ética empresarial y ha desvirtuado sus valores.

\section{Conclusiones}

En las empresas privadas y gubernamentales, y en la sociedad civil de Nicaragua aún hace falta trabajar mucho para desarrollar una adecuada cultura de seguridad, higiene laboral y protección del medio ambiente.

La industrialización ha traído consigo progreso y bienestar, pero también el incremento de los riesgos laborales en los centros de trabajo y la contaminación del medio ambiente. Como resultado, han aparecido problemas globales: destrucción de la capa de ozono, incremento de los gases con efecto invernadero en la atmósfera, calentamiento global y desequilibrio climático, entre otros.

El proceso de globalización, empujado por la búsqueda de nuevos mercados, de materia prima y de los productos terminados de los países industrializados y desarrollados, globalizó los riesgos de contaminación y destrucción del medio ambiente.

El desarrollo económico sostenible, propugnado por los organismos internacionales, considera el respeto a los derechos de las personas y el uso responsable de los recursos naturales.

Los tratados de libre comercio entre países, resultado del avance de la globalización y el empuje de los organismos internacionales, integran en sus cláusulas la seguridad, la higiene laboral y la protección del medio ambiente como condición para participar de estos mercados.

Nicaragua, desde 1945, ha desarrollado su marco legal en relación con la seguridad e higiene laboral. En la década de los noventa, suscribió convenios con distintos organismos internacionales en materia de seguridad, higiene laboral y protección del medio ambiente. Como resultado, el marco jurídico en estas materias ha vivido un gran desarrollo.

Este nuevo marco legal ha fortalecido y creado nuevos entes gubernamentales que conforman la actual estructura normativa, regulatoria y ejecutoria en torno a la seguridad, la higiene laboral y la protección del medio ambiente.

Los tratados de libre comercio y la eliminación de las barreras arancelarias son factores que favorecen a las empresas más competitivas y desfavorecen a las que no lo son.

Actualmente, las empresas nicaragüenses encuentran un entorno en relación a la seguridad, higiene laboral y la protección ambiental que condiciona las fuentes de financiamiento al cumplimiento de las normativas. Igualmente, se exige mayor control de los procesos productivos y de la calidad de los productos terminados, tratamiento de las aguas residuales, de desechos, de emisiones a la atmósfera, cumplimiento de las leyes y regulaciones nacionales e internacionales en relación a estas materias y penaliza la contaminación. 
Un factor de éxito de las empresas es incluir en su visión y misión el aspecto de seguridad, higiene industrial y protección del medio ambiente y tener políticas empresariales acordes con estos valores.

Según estadísticas de las compañías que operan a nivel mundial, de cada diez accidentes, nueve tienen origen en la actitud y el comportamiento humano y solamente uno se debe a las condiciones físicas o factores de trabajo. Este hecho presenta una oportunidad para disminuir los accidentes cuidando los hábitos, los comportamientos y las acciones inseguras.

\section{Recomendaciones}

Una cultura de seguridad, higiene y protección del medio ambiente deberá promoverse en todos los niveles de la organización e integrar los valores empresariales.

Los accionistas y gerentes deben optar por operaciones rentables comprometidas con sus valores, tales como la seguridad, la higiene laboral y protección del medio ambiente. En consecuencia, todas las áreas del negocio deben ser conducidas con ética empresarial. Prioridad gerencial en la gestión de los negocios, debe ser la observancia de las leyes vigentes en Nicaragua en materia laboral y del medio ambiente. Las estrategia empresarial de corto, mediano y largo plazo deberá considerar para su planeación y presupuesto estos temas. Adicionalmente, a esta estrategia debe corresponder una adecuada estructura organizacional.

La planeación estratégica y el presupuesto deben incluir proyectos dedicados a asegurar el cumplimiento de las normas y regulaciones del gobierno de Nicaragua de la materia en cuestión, de acuerdo a los términos y plazos establecidos por la ley y a través de las resoluciones de los entes correspondientes.

En la empresa debe existir un sistema de seguridad, decididamente apoyado por la gerencia que coadyuve al logro de los objetivos.

Las funciones de seguridad, higiene y protección del medio ambiente deben ser parte integral de la organización. La gerencia debe expresar a todos los miembros de la organización, como parte del credo de la empresa, que la seguridad y protección del medio ambiente es una tarea y compromiso de todos.

El sistema de seguridad debe presentar como principal objetivo operar de manera segura y que las personas no sufran accidentes que les causen lesiones o muerte.

El "mercadeo" de la seguridad y la implantación de una nueva cultura de trabajo deben centrarse en los beneficios que obtendrán los trabajadores visualizados como "los compradores".

Dado que el origen del noventa por ciento de los accidentes se debe a actos inseguros, el sistema de seguridad debe dirigirse a la corrección de hábitos inseguros. 
La contabilidad de costos de la empresa debe incluir rubros o centros de costos o cuentas que permitan el análisis de los costos versus beneficio del sistema de seguridad.

\section{Referencias bibliográficas}

-BACA, G. (2000). Evaluación de proyectos, 4ta. edición, México, Ed. Mc. Graw Hill -CHIAVENATO, I., (1998). Administración de Recursos Humanos, 2da. edición, Ed. Mc Graw Hill, Colombia.

-CLEMENT, C. (2000). Economía, Enfoque para América Latina, 4ta. edición, Ed. Mc. Graw Hill, México.

-DENTON, D. (1995). Seguridad Industrial, Administración y Métodos, Ed. Mc Graw Hill, México.

-GOODSTEIN, L.; NOLAN, T. y PFEIFFER (1997). Planeación Estratégica Aplicada, Me Graw Hill, Colombia.

-GRIMALDI, J., ROLLIN, H. (1991). La Seguridad Industrial y su Administración, 2da. edición, Editorial Alfa Omega, México.

-HARRISON, L. (1997). Manual de Auditoría Medioambiental, Higiene y Seguridad, 2da. edición, Mc Graw Hill, México.

-LETAYF, J. y GONZÁLEZ, C. (1994). Seguridad, Higiene y Control Ambiental, Editorial Me Graw Hill, México.

-MINISTERIO DEL AMBIENTE Y LOS RECURSOS NATURALES (MARENA), (2000). Manual sobre regulaciones de calidad ambiental, IMPRIMATUR, Artes Gráficas S.A., Nicaragua.

-MINISTERIO DEL TRABAJO (MITRAB) (2000). Código del Trabajo de la República de Nicaragua, BITECSA, Managua.

-ROMERO, J., (2001) Informe estado del ambiente en Nicaragua 2001, MARENA, Nicaragua.

-SERRANO, A., (2000) Derechoy Ética Empresarial, Apuntes para Maestría en Administración y Dirección de Empresas, Universidad Centroamericana (UCA) Managua. 